\title{
Precorneal Residence Times of Sodium Hyaluronate Solutions Studied by Quantitative Gamma Scintigraphy
}

\author{
G. R. SNIBSON*, J. L. GREAVES $\dagger$, N. D. W. SOPER $\ddagger$ J. I. PRYDAL \\ C. G. WILSON† and A. J. BRON** \\ Oxford, Nottingham and Cambridge
}

\begin{abstract}
Summary
Sodium hyaluronate solutions have been advocated in the management of a variety of dry-eye states. By virtue of their non-Newtonian rheological properties, formulations exhibiting high zero-shear viscosities may be used as an artificial tear with the expectation of prolonged precorneal residence times and improved tolerance. Quantitative gamma scintigraphy was used to evaluate the residence times of $0.2 \%$ and $0.3 \%$ sodium hyaluronate solutions and a polymer-free solution of buffered saline in 12 patients with keratoconjunctivitis sicca and a group of six normal volunteers. Using several indices of residence time, mean values for the sodium hyalure onate solutions were significantly longer than those for buffered saline. Parallel changes in tear film thickness were also demonstrated using a technique based on laser interferometry.
\end{abstract}

Although a variety of therapeutic strategies can be utilised in the management of dry-eye disorders, ${ }^{1,2}$ the basis of therapy remains the artificial tear. The principal limitation of currently available tear substitutes is the short duration of symptom control experienced by some patients. A number of approaches have, therefore, been adopted to prolong the time they remain in contact with the ocular surface including the use of high viscosity formulations, ${ }^{3,4}$ slow-release artificial tears ${ }^{5}$ and occlusion of the lacrimal puncta. ${ }^{6}$ However, none of these methods have met with uniform success.

Polymer solutions formulated at high viscosity have longer residence times, ${ }^{7.8}$ but are poorly tolerated because of transmitted shearing forces associated with blinking and rapid eye movements. It is in this respect that formulations of polymers that display nonNewtonian or pseudoplastic properties offer a potential therapeutic advantage over conventional artificial tears. At concentrations of $0.2 \%$ and $0.3 \%$, sodium hyaluronate solutions exhibit high static, or zero-shear, viscosities but undergo dramatic reduction in viscosity with increasing shear rate" (Fig. 1), thereby offering significantly less resistance to the movement of the lid over the globe than viscous Newtonian formulations. It is the expectation of prolonged residence time associated with high zero-shear viscosities, and of patient acceptance owing to its shear-thinning behaviour, that has lead to the investigation of

*University of Oxford, Department of Ophthalmology.

†University of Nottingham, Department of Physiology and Pharmacology.

†Department of Nuclear Medicine, John Radcliffe Hospital, Oxford.

§University of Cambridge, Department of Physiology

Correspondence to: Professor A. J. Bron, Nuffield Laboratory of Ophthalmology, Walton Street, Oxford OX2 6AW. 


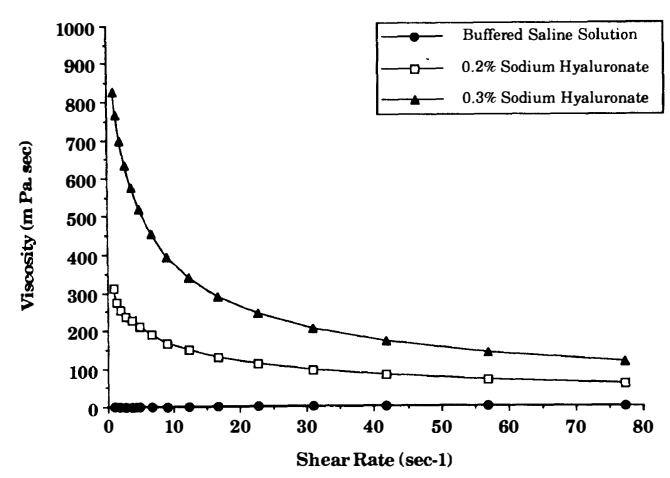

Fig. 1. Change in viscosity of sodium hyaluronate solutions with increasing shear rate. Measurements were made using a Contraves Low-Shear 30 cup and bob viscometer.

sodium hyaluronate solutions as potential tear substitutes.

Few studies have addressed the relationship between the acceptability of viscous eye drops to human subjects and their rheological properties. Methylcellulose and polyvinyl alcohol solutions, which are essentially Newtonian, have been reported to produce discomfort at viscosities of $30 \mathrm{cP}^{10}$ Dudinski et al. evaluated a range of polymers of differing rheology and although unable directly to correlate rheology with acceptability, of those tested, the polymer exhibiting greatest pseudoplasticity was preferred by most subjects to more Newtonian solutions. ${ }^{11}$

Several clinical trials have now reported efficacy of sodium hyaluronate solutions in the treatment of Keratoconjunctivitis sicca and other dry-eye conditions. ${ }^{12.13,14,15,16,17,18}$ Solutions of $0.1 \%$ sodium hyaluronate have also been shown to prolong tear film break-up time ${ }^{18,13,19}$ but few attempts have been made to quantify the precorneal residence times of these formulations in man.

A variety of methods have been devised to quantify residence time. Staining of the conjunctival and corneal epithelium with an Argyrol marker was used by Bach et al. in $1972,{ }^{8}$ and the results obtained indicated that hydroxypropylmethylcellulose (HPMC) had a longer contact time than polyvinyl alcohol. It was concluded that the differences related to the differing viscosities of the polymer solutions. Adler, Maurice and Peterson, in 1971, ${ }^{10}$ reported the use of fluorophotometry to measure the ocular penetration of fluorescein and used these values as an index of the residence time of polymer solutions containing sodium fluorescein. Although greater penetration of fluorescein occurred with viscous solutions, a direct relationship between penetration and viscosity was not demonstrated. Waltman et al. ${ }^{20}$ using a similar method, showed greater penetration from an HPMC vehicle than the less viscous polyvinyl alcohol. Others have used the observable effects of drugs such as pilocarpine, ${ }^{21}$ tropicamide ${ }^{22}$ and homatropine ${ }^{2.3}$ as an index of the residence time of the vehicle. Most of these methods, however, have limitations in that they are indirect, relatively insensitive and reflect variations in other aspects of bioavailability and pharmacological response in addition to considerations of contact time.

Lacrimal gamma scintigraphy, a technique first described by Rossomondo et al. in $1972^{24}$ to evaluate lacrimal drainage, has been used with some modifications in both rabbits ${ }^{25,26}$ and $\operatorname{man}^{27.28}$ to study preocular residence times of drug delivery vehicles. In this study, quantitative gamma scintigraphy was employed to measure the precorneal and ocular surface residence times of two concentrations of sodium hyaluronate and a buffered saline solution in patients with well documented keratoconjunctivitis sicca and in a group of normal volunteers. A method of laser interferometry was also used in an attempt to correlate the effect of these formulations on tear film thickness with residence times.

\section{Method}

Nine females and three males with keratoconjunctivitis sicca or Sjögren's syndrome, aged between 37 and 75 (mean 59 years), were recruited from the External Disease Clinic in Oxford. Each patient was required to have at least two symptoms attributable to dry eyes necessitating regular artificial tear therapy in addition to abnormal values for at least two of the accepted clinical tests of tear film adequacy: Schirmer's test values of less than $5.5 \mathrm{~mm}$ in five minutes and tear film break-up times, using a non-invasive method, ${ }^{29}$ of less than ten seconds were regarded as abnormal. 
Rose bengal staining of the interpalpebral region of the ocular surface was graded on a scale of $0-5$ for the cornea and each of the medial and lateral bulbar conjunctival areas and an aggregate score of six or more was determined to be abnormal. None of the patients gave a history of other external disease or anterior segment surgery, nor were they currently using any form of topical medication other than artificial tears. A group of six normal volunteers, without external disease and of a similar age and sex distribution, also participated in the study. Each subject was given a verbal and written explanation of the procedure and their informed consent was obtained.

Three formulations were studied: $0.2 \%$ and
$0.3 \%$ sodium hyaluronate $(\mathrm{SH})$ in a buffered saline solution and the buffered saline itself (BS). Each subject was randomly allocated to receive the three formulations in one of six possible orders and studies were performed a minimum of five days apart to eliminate any possibility of interaction or residual activity. The isotope used was technetium $99 \mathrm{~m}$ chelated with diethylenetriaminepentaacetic acid (DTPA), a commonly employed radiopharmaceutical, in a sterile saline solution. A small volume $(0.10-0.40 \mathrm{ml})$ of each formulation was replaced with the isotope preparation, the exact volume depending on the activity of the ${ }^{99} \mathrm{Tc}^{\mathrm{m}}$ eluted from the generator at the time of the study. The mean dilution was $4 \%$ and was similar for the three for-

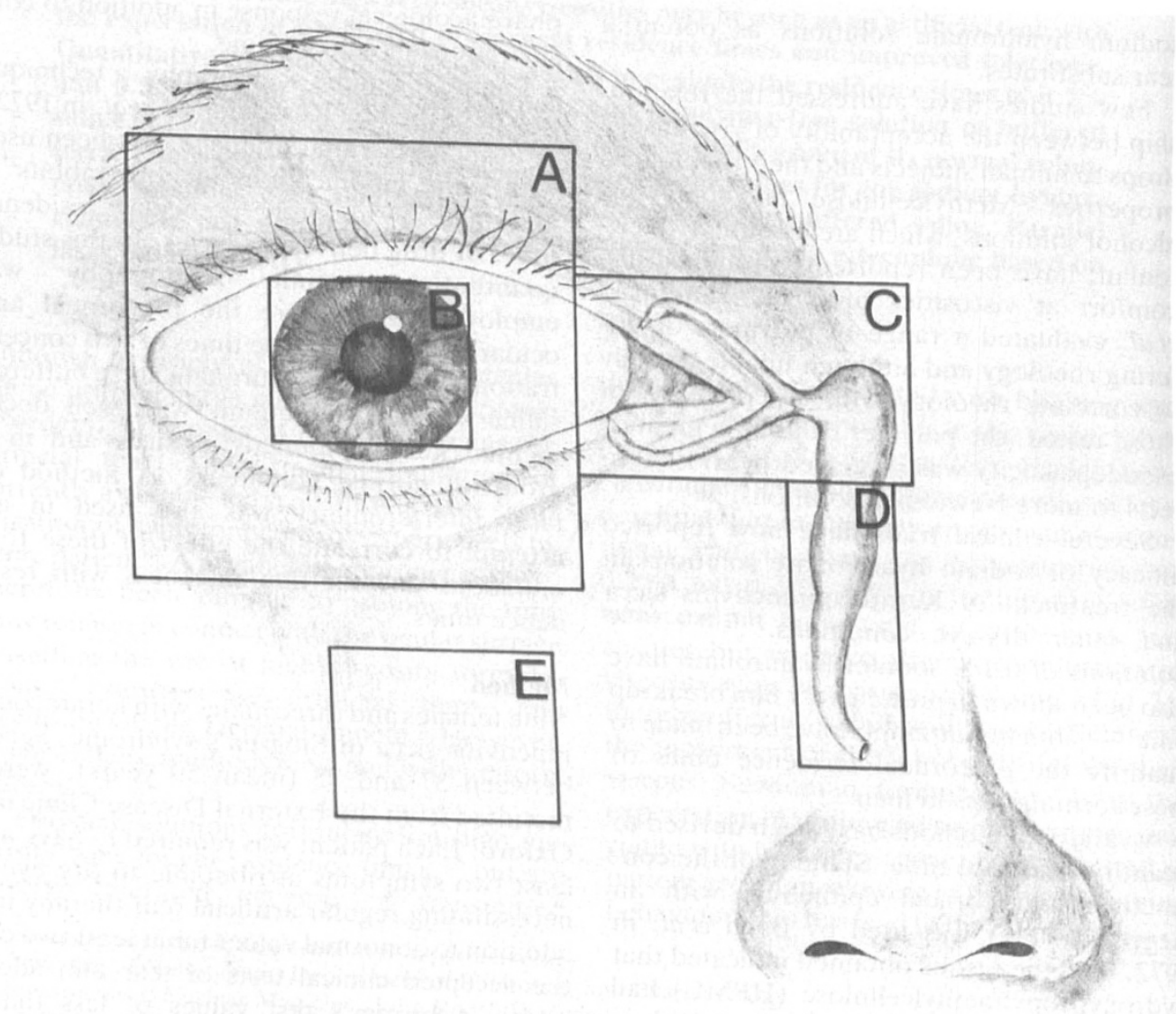

Fig. 2. Regions of interest (ROI). 
mulations studied. Sufficient tracer was added to give each $25 \mu \mathrm{l}$ drop an activity of approximately one megaBecquerel (MBq). The resultant activity of the individual drop was

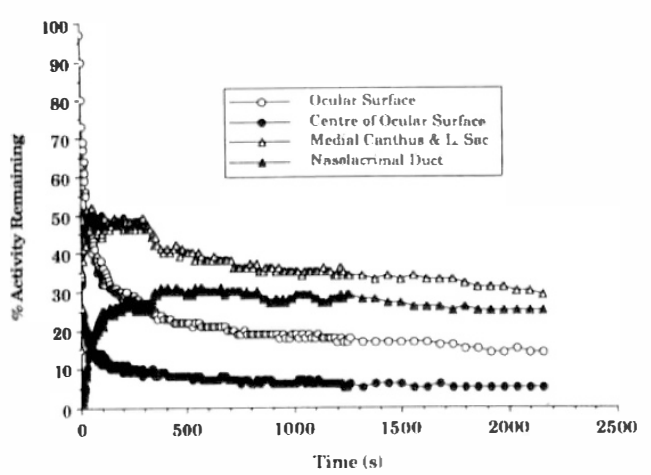

Fig. 3a.

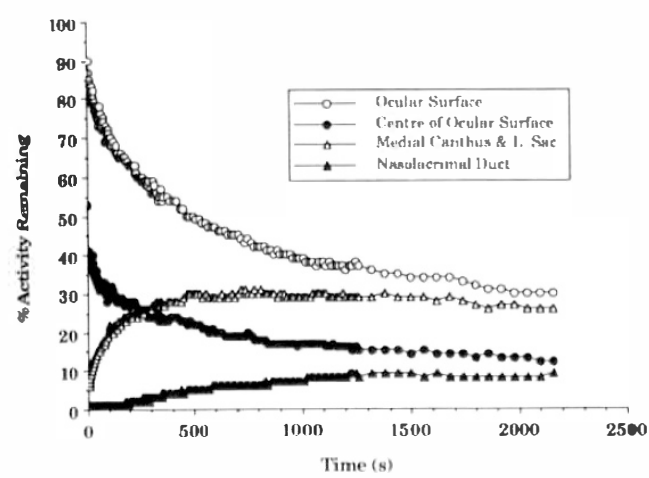

Fig. 3h.

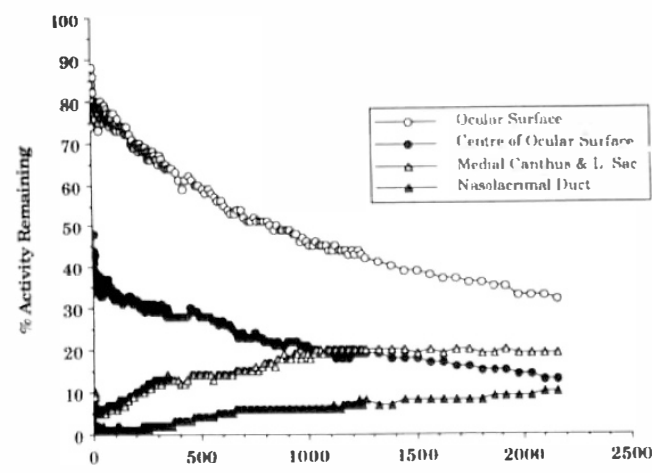

Fig. 3c.

Fig. 3. Clearance of the test formulations from four regions of interest in dry-eye patients (mean data, $n=12$ ): (a) Buffered saline solution; (b) $0.2 \%$ sodium hyaluronate; (c) $0.3 \%$ sodium hyaluronate. calculated from the measured activity of $5.0 \mathrm{ml}$ of the labelled formulation immediately prior to commencement of each study. The mean activity was $1.0 \mathrm{MBq}$ (range 0.81 1.55) without significant differences between the three formulations. The reliability and accuracy of these steps were confirmed in separate experiments in which multiple samples were taken from a range of labelled solutions and counts obtained from each sample with the gamma camera under experimental conditions.

Subjects were seated with their head supported so that the eye to be studied was $5.0 \mathrm{~cm}$ from the $3.5 \mathrm{~mm}$ aperture of the pinhole collimator of the IGE 400T gamma camera. A $25 \mu \mathrm{l}$ drop was placed on the superior aspect of the globe using a Transferpettor direct displacement pipette and the subject was encouraged to maintain a normal blink rate with both eyes open and in the primary position. Acquisition was commenced simultaneously and continued without interruption for $36 \mathrm{~min}$ utes. In order to detect early and rapid changes, time frames of two seconds were used for the first two minutes increasing in length to 60 second frames for the last $15 \mathrm{~min}$ utes. Images were stored on magnetic disc and processed by a Picker PCS 512 minicomputer and later copied to optical disc for permanent storage.

At the conclusion of each study, the field of view of the gamma camera was divided into five regions of interest (ROI) on the basis of previously determined anatomical landmarks and the distribution of activity seen when the image of the totalised study is displayed on the monitor. One large rectangle was used to encompass the entire ocular surface excluding the region of the inner canthus. A smaller rectangle was used to represent the corneal surface excluding activity associated with the tear meniscus and any possible contamination of the lashes or lid margin. The medial canthal area and the lacrimal sac were described by a third region and a fourth outlined the nasolacrimal duct. A small peripheral area was used to record background gamma radiation (Fig. 2).

In a separate experiment, the effect of each formulation on tear film thickness was studied dynamically in a normal volunteer using a 


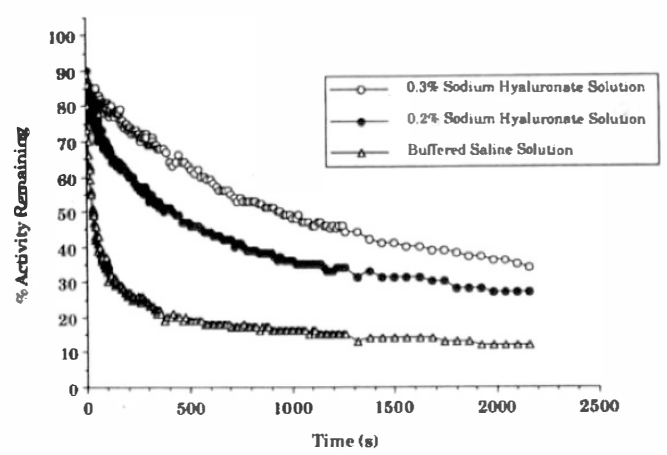

Fig. 4a.

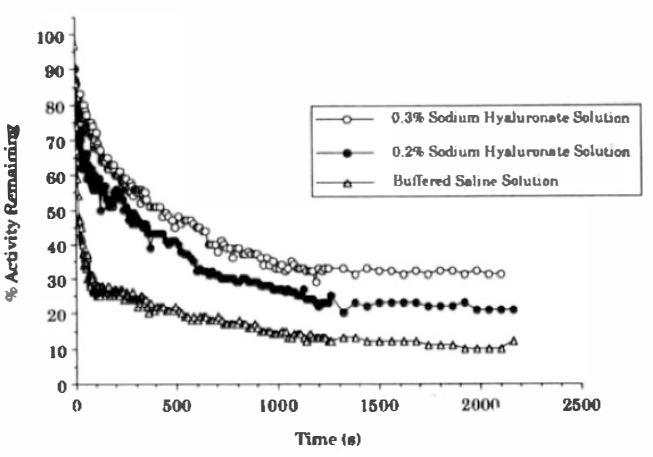

Fig. th.

Fig. 4. Ocular surface clearance curves for the three formulations studied (mean data): (a) Dry-eye patients $(n=12)$; (b) Normal subjects $(n=5)$.

technique based on laser interferometry. Baseline measurements were made prior to instillation of a $10 \mu \mathrm{l}$ drop and interference fringes were recorded and measurements made until tear film thickness returned to baseline. Each solution was studied three times on separate days. A detailed account of the method used is reported elsewhere. ${ }^{30}$

\section{Results}

Counts were obtained from each region of interest and for each of the 183 time frames. From these counts, after correction for background values, curves were generated expressing the percentage activity remaining as a function of time for each of the regions of interest (Fig. 3). Curve-fitting was performed using the programme MINIM for the Apple Macintosh computer. In this way rates of clearance of the isotope from the cornea and ocular surface could be compared for each test solution in each of the 18 subjects (Fig. 4). Due to excessive movement during the study of one normal subject, curve-fitting was not possible and these data were excluded from further analysis.

From individual curve-fit plots for the dryeye patients, the time taken for half of the formulation (reflected by the detected gamma emission from the $\left.{ }^{99} \mathrm{Tc}^{\mathrm{m}}\right)$ to be cleared from the ocular surface $\left(T_{50}\right)$ was derived for each solution. Student's paired $t$-test was used to evaluate differences between mean $T_{50}$ values for the three formulations studied. The $0.3 \%$ solution of sodium hyaluronate cleared sig- nificantly more slowly than the buffered saline solution $(\mathrm{p}<0.001)$. The $0.2 \%$ solution also remained on the ocular surface much longer than the polymer-free preparation $(p=0.003)$. The more viscous $0.3 \%$ solution cleared more slowly than $0.2 \%$ sodium hyaluronate and this difference was also significant $(p=0.033)$. Similarly, clearance of the sodium hyaluronate solutions from the central corneal surface was also retardeḍ compared with the buffered saline. Corresponding increases in activity recorded from the areas of the lacrimal sac and nasolacrimal duct were much smaller and slower for the more viscous formulations.

Although mean $T_{50}$ values for the normal subjects followed the same trend, there was considerable variation between subjects and the differences did not attain statistical significance. Mean $T_{50}$ values for the hyaluronate solutions were approximately half those found in dry eye patients (Fig. 5).

The function which gave closest approximation to the clearance curves in most cases was the following biexponential equation: $\mathrm{y}=\mathrm{A}^{*} \exp \left(-\mathrm{k}_{1} \mathrm{t}\right)+\mathrm{B}^{*} \exp \left(-\mathrm{k}_{2} \mathrm{t}\right)$. This provides constants, $k_{1}$ and $k_{2}$, which allow comparisons between solutions for different phases of the curves. Using analysis of variance, significant differences between mean values for both $k_{1}$ and $k_{2}$ for the three formulations were demonstrated for the ocular surface curves. This was the case in both normal subjects and those with dry eyes

The area under the curves provides another 


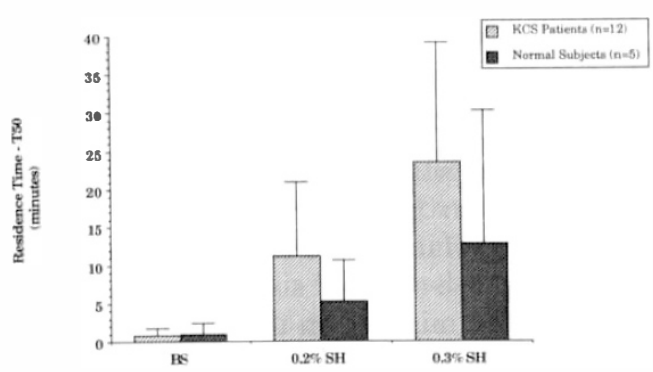

Fig. 5. Mean ocular surface $T_{50}$ values and standard deviations for buffered saline (BS), $0.2 \%$ and $0.3 \%$ sodium hyaluronate $(\mathrm{SH})$ solutions.

index of residence time and the differences between mean areas for the ocular surface region were also significant in favour of the more viscous formulations (between BS and $0.2 \% \mathrm{SH}, \mathrm{p}=0.006$; between $\mathrm{BS}$ and $0.3 \% \mathrm{SH}, \mathrm{p}<0.001)$.

The volume of the solution remaining at the end of the study provides some indication of its long term persistence. By comparing counts obtained from the 35 th minute of acquisition, after correction for initial activity, sodium hyaluronate would appear to persist on the ocular surface in much greater quantities than the polymer-free buffered saline.

Tear film thickness, in all cases, increased after instillation of the drop and reached a maximum value within a few seconds. Maximum thickness was noted, as was the time taken for the tear film to return to its baseline thickness (Fig. 6). There appears to be a clear trend with greater thickening produced by the sodium hyaluronate solutions; the difference between the thickening effects of the buffered saline and $0.2 \%$ sodium hyaluronate being statistically significant $(\mathrm{p}=0.026)$. Although recovery time would also appear to be viscosity-related, further experiments are necessary to confirm this.

\section{Discussion}

A variety of properties have been regarded as important in the formulation of tear substitutes including viscosity, surface tension, tonicity, $\mathrm{pH}$, and the ability to simulate the properties of tear film mucus. Clinical trials to date have failed to give preeminence to any one of these factors and the search for the ideal artificial tear continues. A feature of the tear film attributed to ocular surface mucus ${ }^{31}$ is the psuedoplastic property of shear rate dependent viscosity, a property shared by sodium hyaluronate in solution by virtue of its high molecular weight and flexible, open-coil conformation. ${ }^{9}$ The possible advantage of an artificial tear possessing these characteristics is the opportunity to use high viscosity formulations with prolongation of precorneal residence time and tear film stability. To our knowledge, the residence times of sodium hyaluronate solutions in dry eye patients have not previously been studied.

Quantitative gamma scintigraphy is a safe and effective non-invasive means of evaluating the preocular residence times of ophthalmic preparations. ${ }^{32}$ Using this method we were able to demonstrate, in dry-eye patients, a mean ocular surface residence time for $0.3 \% \mathrm{SH}$, as reflected by the mean $\mathrm{T}_{50}$, of 23.5 minutes $(1,407 \mathrm{sec})$. For $0.2 \% \mathrm{SH}$ the mean $\mathrm{T}_{50}$ was 11.1 minutes $(665 \mathrm{sec})$ compared with less than one minute $(50 \mathrm{sec})$ for the buffered saline solution. Despite marked variation in rate of clearance between subjects all these differences attained statistical significance. Similar trends were observed for the region corresponding to the corneal surface. As would be anticipated, the appearance of the tracer in the lacrimal sac and nasolacrimal duct was delayed and slower for the viscous preparations. Delayed drainage would be expected to enhance the persistence of the polymer in the tear film meniscus which could act as a reservoir serving to replenish the preocular tear/polymer film with each blink.

Although early clearance kinetics are of interest, only a small proportion of the instilled volume is likely to be necessary to

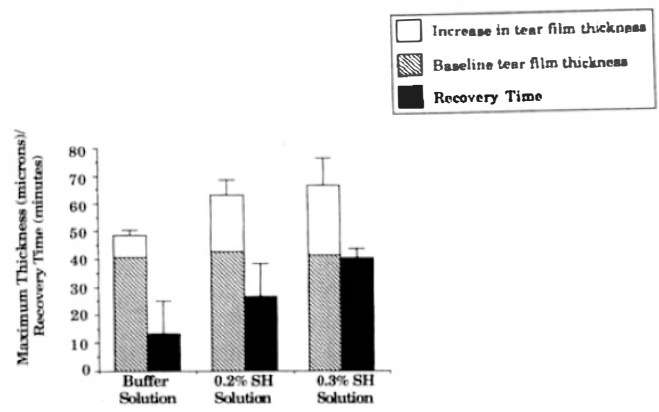

Fig. 6. Maximum tear film thickness and recovery time after instillation of buffered saline (BS), $0.2 \%$ and $0.3 \%$ sodium hyaluronate $(\mathrm{SH})$ solutions. 
provide stability to the tear film. An indication of the longer term persistence of small quantities of polymer is given by the second phase of the biexponential curve described by the constant $k_{2}$. Differences were also demonstrated on the basis of $\mathrm{k}_{2}$ in favour of the more viscous formulations. Similarly, by comparing areas under clearance curves and corrected counts obtained from the penultimate frames (35th minute), greater persistence of the $0.3 \%$ and $0.2 \%$ sodium hyaluronate can be demonstrated. At least in the case of sodium hyaluronate solutions, these findings would support the statement that residence time is viscosity-related, a concept that has been challenged in the past. ${ }^{10}$. These data also compare favourably with the residence times of polyvinyl alcohol, hydroxypropylmethylcellulose $^{28}$ and hydroxyethylcellulose ${ }^{33}$ that have been determined using similar scintigraphic methods.

The shorter residence times of sodium hyaluronate which we have demonstrated in normal subjects, compared with patients with $\mathrm{KCS}$, would caution against comparing residence times determined in these different populations and suggest that it is more appropriate to study residence times of artificial tears in individuals with dry eyes. This difference might be explained solely by the dilutional and wash-out effects of reflex and basal lacrimation which are diminished in KCS; but squamous metaplasia, which is evident to some extent in severely dry eyes ${ }^{34}$ may also play a role in altering adhesion of the polymer or increasing the resistance to canalicular outflow. Alternatively, an alteration of tear mucin or of surface epithelial glycocalyx in dry eyes might modify the interaction with sodium hyaluronate and explain the differences in group behaviour. The rate of clearance of the buffered saline was similar for both groups of subjects and we were unable to confirm the findings of Weber et al. ${ }^{35}$ who demonstrated hyperexcretion in a subgroup of dry-eye patients.

Viscosity alone may to a large extent explain the prolonged residence times of sodium hyaluronate solutions, but adhesion or physical attachment to the ocular surface has been suggested by some ${ }^{12,36}$ and has been recently demonstrated using an in vitro experimental model. ${ }^{37}$ Although the presence of binding sites for sodium hyaluronate has been demonstrated on the surface of the corneal endothelium, none has been found on the epithelial surface. ${ }^{38}$ Scanning and transmission electron microscopy has been employed by Hazlett and Barrett ${ }^{39}$ to demonstrate the persistence of sodium hyaluronate for at least 60 minutes on the corneal surface of mice that would appear not to be dependent on the presence of an intact mucus layer. This would suggest an interaction between the polymer and either the glycocalyx or the cell membranes of the epithelial surface.

Thickening of the precorneal tear film by viscous polymer solutions has been demonstrated previously using a fluorophotometric method $;^{40}$ however, the duration of this effect has not been quantified. Using a non-invasive method, we were able to demonstrate thickening of the tear film after instillation of all three test formulations. The magnitude of this effect appears to be viscosity-related; and, for the hyaluronate solutions, the recovery time is approximately twice the ocular surface $T_{50}$ value.

One mechanism by which this thickening might occur is by increasing the volume of the marginal tear meniscus. It is also likely that a large hydrophilic molecule adsorbed at the corneal surface would be capable of stabilising to some extent the aqueous phase into which it projects. A third mechanism, suggested and supported experimentally by Benedetto, Shah and Kaufman, ${ }^{40}$ is that a film of polymer adsorbed at the air/tear interface is able to support a layer of water beneath it which is dragged in over the ocular surface and each blink.

The clinical value of sodium hyaluronate solutions in the management of patients with dry eyes has yet to be determined conclusively. The design and conduct of sound clinical trials of artificial tears are not without difficulty, owing to the paucity of reliable objective parameters for assessing change. These studies must also be of sufficient power to account for the therapeutic effect of the saline placebo. Some (unpublished) randomised controlled trials have failed to demonstrate a clear benefit over placebo. However, Nelson and Farris have shown $0.1 \%$ sodium 
hyaluronate to be at least as effective as $1.4 \%$ polyvinyl alcohol ${ }^{18}$ and Sand, Marner and Norn have recently reported a placebo-controlled trial with differences in rose bengal staining, tear film break-up time and patient preference in favour of a $0.2 \%$ sodium hyaluronate preparation. ${ }^{41} \mathrm{~A}$ number of uncontrolled clinical trials in selected patients have also reported efficacy. ${ }^{12,13,14}$

The properties of artificial tear formulations that are most important in the enhancement of tear film thickness and stability remain, to a large extent, unknown. However, in the light of some encouraging clinical data, the prolonged residence times of sodium hyaluronate solutions together with their favourable rheological properties, would suggest that their further evaluation as a tear substitute may provide a useful new agent in the management of dry-eye states.

The sodium hyaluronate solutions were provided by Pharmacia Ophthalmics AB, Uppsala, Sweden. The authors have no commercial interest in any of the products mentioned. We are grateful to Dr. B. Shepstone and the staff of the Nuclear Medicine Department, John Radcliffe Hospital, Oxford, for their helpful cooperation.

\section{References}

${ }^{1}$ Bron AJ: Duke-Elder Lecture: Prospects for the dry eye. Trans Ophthalmol Soc UK 1985, 104: 801-26.

${ }^{2}$ Lemp MA: General measures in management of the dry eye. Int Ophthalmol Clin 1987, 27: 36-43.

${ }^{3}$ Blaug SM and Canada AT: Relationship of viscosity, contact time, and prolongation of action of methylcellulose-containing ophthalmic solutions. Am J Hosp Pharm 1965, 22: 662-6.

${ }^{4}$ Wilson CG, Olejnik O, Hardy JG: Precorneal drainage of polyvinyl alcohol solutions in the rabbit assessed by gamma scintigraphy. J Pharm Pharmacol 1986, 35: 451.

${ }^{5}$ Werblin TP, Rheinstrom SD, Kaufman HE: The use of slow-release artificial tears int he long-term management of keratitis sicca. Ophthalmology 1981, 88(1): 78-81.

${ }^{6}$ Dohlman $\mathrm{CH}$ : Punctal occlusion in keratoconjunctivitis sicca. Trans ophthalmol Soc UK 1978, 98: 1277-81.

${ }^{7}$ Chrai SS and Robinson JR: Ocular evaluation of methylcellulose vehicle in albino rabbits. J Pharm Sci 1974, 63: 1218.

${ }^{8}$ Bach FC, Adam JB, McWhirter HC, Johnson JE: Ocular retention of artificial tear solutions. Ann Ophthalmol 1972, 4: 116-19.

${ }^{9}$ Bothner $\mathrm{H}$ and Wik O: Rheology of intraocular fluids. In Rosen ES ed. Viscoelastic Materials: Basic science and clinical applications. Pergamon Press, 1989: 3-22.
${ }^{10}$ Adler CA, Maurice DM, Paterson ME: The effect of viscosity of the vehicle on the penetration of fluorescein into the human eye. Exp Eye Res 1971, 11: $34-42$.

${ }^{11}$ Dudinski O, Finnin BC, Reed BL: Acceptability of thickened eye drops to human subjects. Curr Ther Res 1983, 33(2): 322-37.

${ }^{12}$ Polack FM and McNiece MT: The treatment of dry eyes with $\mathrm{Na}$ hyaluronate (Healon): a preliminary report. Cornea 1982, 1: 133-36.

${ }^{13}$ DeLuise VP and Peterson WS: The use of topical Healon tears in the management of refractory dry-eye syndrome. Ann Ophthalmol 1984, 16: 823-4.

${ }^{14}$ Stuart JC and Linn JG: Dilute sodium hyaluronate (Healon) in the treatment of ocular surface disorders. Ann Ophthalmol 1985, 17: 190-92.

${ }^{15}$ Gee S and Tabbara KF: The effects of hyaluronic acid on patients with keratoconjunctivitis sicca. Saudi Bull Ophthalmol 1987, 2(3): 24-6.

${ }^{16}$ Limberg MB, McCaa C, Kissling GE, Kaufman HE: Topical application of hyaluronic acid and chondroitin sulfate in the treatment of dry eyes. $A m \mathrm{~J}$ Ophthalmol 1987, 103: 194-7.

${ }^{17}$ Laflamme MY and Swieca R: A comparative study of two preservative-free tear substitutes in the management of severe dry eye. Can J Ophthalmol 1988, 23: 174-6.

${ }^{18}$ Nelson JD and Farris RL: Sodium hyaluronate and polyvinyl alcohol artificial tear preparations: a comparison in patients with keratoconjunctivitis sicca. Arch Ophthalmol 1988, 106: 484-7.

${ }^{19}$ Mengher LS, Pandher KS, Bron AJ: Value of sodium hyaluronate $(0.1 \%$ Healon $)$ in the treatment of dry eyes (Abstract). Ophthal Res 1986, 18: 140 .

${ }^{20}$ Waltman SR and Patrowicz TC: The effects of hydroxypropyl methylcellulose and polyvinyl alcohol on intraocular penetration of topical fluorescein in man. Invest Ophthalmol 1970, 9: 966-70.

${ }^{21}$ Haas JS and Merril DL: The effect of methylcellulose on responses to solutions of pilocarpine. $A m$ J Ophthalmol 1962, 54: 2.

2 Saettone MF, Giannacinni B, Savigni P, Wirth A: The effect of different ophthalmic vehicles on the activity of tropicamide in man. J Pharm Pharmac 1980, 32: 519-21.

${ }^{23}$ Mueller WH and Deardorff DL: Ophthalmic vehicles: the effect of methylcellulose on the penetration of homatropine hydrobromide through the cornea. J Am Pharm Assoc 1956, 45: 334-41.

${ }^{24}$ Rossomondo RM, Carlton WH, Trueblood JH, Thomas RP: A new method of evaluating lacrimal drainage. Arch Ophthalmol 1972, 88: 532-5.

${ }^{25}$ Fitzgerald P, Hadgraft J, Kreuter J, Wilson CG: A gamma scintigraphic evaluation of microparticulate ophthalmic delivery systems: liposomes and nanoparticles. Int J Pharm 1987, 40: 81-4.

${ }^{26}$ Zaki I, Fitzgerald P, Hardy JG, Wilson CG: A comparison of the effect of viscosity on the precorneal residence of solutions in rabbit and man. J Pharm Pharmacol 1986, 38: 463-66.

${ }^{27}$ Chrai SS, Patton AM, Mehta A, Robinson JR: Lacrimal and instilled fluid dynamics in rabbit eyes. $J$ Pharm Sci 1973, 62: 1112-21. 
${ }^{28}$ Trueblood JH, Rossomondo RM, Carlton WH, Wilson LA: Corneal contact times of ophthalmic vehicles: evaluation by microscintigraphy. Arch Ophthalmol 1975, 93: 127-30.

${ }^{29}$ Mengher LS, Bron AJ, Tonge SR, Gilbert DJ: Noninvasive assessment of tear film stability. In Holly FJ ed. The preocular tear film in health, disease, and contact lens wear. Lubbock, Texas. 1986: 663-69.

${ }^{30}$ Prydal JI: Dynamic time resolution of human tear film thickness. Paper presented at the 1989 Oxford Ophthalmological Congress. (Unpublished.)

${ }^{31}$ Kaura R and Tiffany $\mathrm{J}$ : The role of mucous glycoproteins in the tear film. In Holly FJ ed. The preocular tear film in health, disease, and contact lens wear. Lubbock, Texas. 1986: 728-32.

${ }^{32}$ Wilson CG: Scintigraphic evaluation of polymeric formulations for ophthalmic use. In Saettone MS, Bucci G, Speiser P eds. Ophthalmic drug delivery. Biopharmaceutical, technological and clinical aspects. Fidia Research Series, Liviana Press, Padova, 1987, 11: 141-50.

${ }^{33}$ Wilson CG, Olejnik O, Greaves JL, Kennedy D: Animal models for studying the precorneal residence of ophthalmic solutions (abstract). ARVO Supplement Invest Ophthalmol Vis Sci 1989, 30: 247.

${ }^{34}$ Nelson JD: Impression Cytology. Cornea 1988, 7: 71-81.
${ }^{35}$ Weber P, Lubkin V, Kramer P, Liebeskind A, McKelvey W: Dacryoscintigraphy to determine lacrimal outflow rates in normal versus dry eye patients. ARVO Abstracts 1987, 158.

${ }^{36}$ Gurny R, Ibrahim H, Aebi A, Buri P, Wilson CG, Washington N, Edman P, Camber O: Design and evaluation of controlled release systems for the eye. Journal of Controlled Release 1987, 6: 367-73.

${ }^{37}$ Saettone MF, Chetoni P, Torracca MT, Burgalassi S, Giannaccini B: Evaluation of muco-adhesive properties and in vivo activity of ophthalmic vehicles based on hyaluronic acid. Int J Pharm 1989, 51: 203-12.

${ }^{38}$ Madsen K, Stenevi U, Apple DJ, Härfstrand A: Histochemical and receptor binding studies of hyaluronic acid and hyaluronic acid binding sites on corneal endothelium. Ophthalmic Practice 1989, 7: 2-8.

${ }^{39}$ Hazlett LD and Barrett R: Sodium hyaluronate eye drop. A scanning and transmissioin electron microscopy study of the corneal surface. Ophthal Res 1987, 19: 277-84.

${ }^{40}$ Benedetto DA, Shah DO, Kaufman HE: The instilled fluid dynamics and surface chemistry of polymers in the preocular tear film. Invest Ophthalmol 1975, 14: 887-902.

${ }^{41}$ Sand BB, Marner K, Norn MS: Sodium hyaluronate in the treatment of keratoconjunctivitis sicca. Acta Ophthalmol 1989, 67: 181-3. 